Article

\title{
Simulation Study on the Influence of Multifrequency Ultrasound on Transient Cavitation Threshold in Different Media
}

\author{
Hu Dong ${ }^{1,2}$, Xiao Zou ${ }^{1}$ (D) and Shengyou Qian ${ }^{1, *}$ \\ 1 School of Physics and Electronics, Hunan Normal University, Changsha 410081, China; \\ wjd3203@smail.hunnu.edu.cn (H.D.); shawner@hunnu.edu.cn (X.Z.) \\ 2 School of Information Science and Engineering, Changsha Normal University, Changsha 410100, China \\ * Correspondence: shyqian@hunnu.edu.cn
}

Received: 25 May 2020; Accepted: 9 July 2020; Published: 11 July 2020

check for updates

Featured Application: This work provides a theoretical basis for how to optimize the combination of multifrequency ultrasound to achieve better reduction of the transient cavitation threshold in the medium and provide guidance of its applications for tissue ablation.

\begin{abstract}
Through the introduction of multifrequency ultrasound technology, remarkable results have been achieved in tissue ablation and other aspects. By using the nonlinear dynamic equation of spherical bubble, the effects of the combination mode of multifrequency ultrasound, the peak negative pressure and its duration, the phase angle difference, and the polytropic index on the transient cavitation threshold in four different media of water, blood, brain, and liver are simulated and analyzed. The simulation results show that under the same frequency difference and initial bubble radius, the transient cavitation threshold of the high-frequency, triple-frequency combination is higher than that of the low-frequency, triple-frequency combination. When the lowest frequency of triple frequencies is the same, the larger the frequency difference, the higher the transient cavitation threshold. When the initial bubble radius is small, the frequency difference has little effect on the transient cavitation threshold of the triple-frequency combination. With the increase of initial bubble radius, the influence of frequency difference on the transient cavitation threshold of the higher frequency combination of triple frequency is more obvious than that of the lower frequency combination of triple frequency. When the duration of peak negative pressure or peak negative pressure of the multifrequency combined ultrasound is longer than that of the single-frequency ultrasound, the transient cavitation threshold of the multifrequency combined ultrasound is lower than that of the single-frequency ultrasound; on the contrary, the transient cavitation threshold of the multifrequency combined ultrasound is higher than that of the single-frequency ultrasound. When the phase angle difference of multifrequency excitation is zero, the corresponding transient cavitation threshold is the lowest, while the change of the polytropic index has almost no effect on the transient cavitation threshold for the multifrequency combination. The research results can provide a reference for multifrequency ultrasound to reduce the transient cavitation threshold, which is of great significance for the practical application of cavitation.
\end{abstract}

Keywords: bubble; nonlinear; multifrequency combination; transient cavitation threshold

\section{Introduction}

Cavitation is a process of bubble oscillation and rupture in the medium under ultrasonic irradiation. Bubbles can either oscillate stably, that is, stable cavitation, or expand gradually and finally collapse, that is, transient cavitation [1]. Generally speaking, cavitation refers to transient cavitation, and the 
generation of transient cavitation is affected by many factors, including ultrasonic frequency, bubble size, and characteristics of the surrounding medium [2]. It is widely used in the treatment of tumor and thrombus in the medical field to produce high temperature and pressure [3-5]. Compared with traditional single-frequency ultrasound, multifrequency ultrasound has unique advantages in enhancing the cavitation effect, such as reducing cavitation threshold and improving reaction efficiency [6-8].

In order to study the cavitation effect of multifrequency ultrasound, many experiments have been carried out. Guo et al. [6] and Ma et al. [9] found that when the dual-frequency high-intensity focused ultrasound (HIFU) transducer with different frequencies was used to ablate chicken, compared with the single-frequency HIFU alone, the dual-frequency irradiation could obtain faster temperature rise. He et al. [10] used transducers with ultrasonic frequencies of $1563 \mathrm{MHz}$ and $1573 \mathrm{MHz}$ to irradiate the target porcine liver tissue at the same time, and carried out dual-frequency tissue ablation experiments. The results showed that under the same power condition, dual-frequency HIFU could produce greater lesions than traditional single-frequency HIFU, which may be the result of dual-frequency ultrasonic cavitation enhancement. When Gilles et al. [11] superposed two sine wave signals with ultrasonic frequencies of $531 \mathrm{kHz}$ and $565 \mathrm{kHz}$ for irradiation, compared with the single-frequency stimulation of $552 \mathrm{kHz}$, the transient cavitation threshold was reduced by nearly $30 \%$. Saletes et al. [12] tested the thrombolytic efficiency of dual-frequency excitation composed of $535 \mathrm{kHz}$ and $565 \mathrm{kHz}$ on the blood clot in vitro and compared with the thrombolytic efficiency of single-frequency excitation of $550 \mathrm{kHz}$ with the same power. The dual-frequency excitation doubled the thrombolytic efficiency and dissolved a $0.8 \mathrm{~cm}^{3}$ blood clot in $5 \mathrm{~min}$. Suo et al. [13] used $1.45 \mathrm{MHz}$ and $1.5 \mathrm{MHz}$ dual-frequency ultrasound to achieve the same ablation efficiency under the same acoustic power and excitation conditions and found that, compared with single-frequency ablation, dual-frequency ultrasound could save about $30 \%$ power. Sasaki et al. [14] superposed $0.8 \mathrm{MHz}$ and $1.6 \mathrm{MHz}$ ultrasound to irradiate biological tissues, which confirmed that dual-frequency HIFU irradiation was more effective in enhancing tissue coagulative necrosis volume. Feng et al. [15] found that, compared with dual-frequency HIFU irradiation, triple-frequency $(28 \mathrm{kHz}, 1 \mathrm{MHz}$, and $1.87 \mathrm{MHz})$ irradiation could further improve cavitation efficiency. In the study of temperature rise of tissue ablation, under the same irradiation power and time, Guo et al. [16] used any two frequencies of $0.95 \mathrm{MHz}, 1.5 \mathrm{MHz}$, and $3.3 \mathrm{MHz}$ for dual-frequency combined irradiation. Compared with the combination of triple-frequencies of $0.95 \mathrm{MHz}, 1.5 \mathrm{MHz}$, and $3.3 \mathrm{MHz}$, under the same excitation conditions, the temperature rise of the triple-frequency combination irradiation was higher than that of the dual-frequency combination. Many experimental results show that the cavitation effect can be significantly enhanced by the combination of multifrequency ultrasound irradiation, but there are few theoretical studies on this problem. On the one hand, although some unique characteristics have been found in some preliminary studies of cavitation, the mechanism of multifrequency combination to enhance bubble cavitation has not been fully understood, and on the other hand, the combination of multifrequency ultrasound has an impact on the transient cavitation threshold in the medium. The transient cavitation threshold has potential application value in contrast imaging, therapeutic drug development, and evaluation of localized effects on the body [17]. Theoretically, this paper discusses how to optimize the combination of multifrequency ultrasound to achieve better reduction of the transient cavitation threshold in the medium, and provides evidence for the enhancement of cavitation effect by multifrequency ultrasound, which has important guiding significance for practical application, such as multifrequency thermal ablation and so forth.

In this paper, the transient cavitation threshold of water, blood, brain, and liver are calculated by solving the bubble dynamic equation. The effects of the combination mode of multifrequency ultrasound, the peak negative pressure and its duration, the phase angle difference, and the polytropic index on the transient cavitation threshold of different media are studied. 


\section{Principle and Method}

The numerical simulation of the transient bubble radial response is carried out by using the Yang-Church model [18], which considers the influence of medium viscosity on the vibration of a single microbubble. The vibration of a spherical bubble in an infinite range of incompressible thermoviscous medium is solved by the Keller-Miksis dynamic equation $[19,20]$.

$$
\left(1-\frac{\dot{R}}{c}\right) R \ddot{R}+\frac{3}{2}\left(1-\frac{\dot{R}}{3 c}\right) \dot{R^{2}}=\left(1+\frac{\dot{R}}{c}\right) \frac{P}{\rho}+\frac{R}{\rho c} \frac{d P}{d t}
$$

where $R$ is the bubble radius, $\dot{R}$ is the bubble wall velocity, $\ddot{R}$ is the bubble wall acceleration, $c$ is the sound velocity in the surrounding medium, $\rho$ is the density of the surrounding medium. The bubble pressure $P$ can be expressed as follows [21]:

$$
P=p_{g}-p_{0}-\frac{2 \sigma}{R}+3 \int_{R}^{\infty} \frac{\tau_{r r}}{r} d r+p_{a}
$$

where $p_{g}$ is the gas pressure in the bubble, $p_{0}$ is the ambient pressure, $p_{a}$ is the excitation signal sound pressure, $\sigma$ is the surface tension, $\tau_{r r}$ is the shear stress, $r$ is the radial radius of the bubble. The gas pressure $p_{g}$ in the bubble can be expressed as follows [22]:

$$
p_{g}=p_{g 0}\left(R_{0} / R\right)^{3 \kappa}
$$

where $R_{0}$ is the initial bubble radius, $\kappa$ is the polytropic index, which is determined by the pressure and temperature in the bubble, reflecting the relationship between the air density and the pressure change in the bubble. $p_{g 0}$ is the initial gas pressure in the bubble, defined as:

$$
p_{g 0}=p_{0}+2 \sigma / R_{0}
$$

The shear stress can be expressed as [23]:

$$
\tau_{r r}=2\left(G \gamma_{r r}+\mu \dot{\gamma}_{r r}\right)
$$

where $\gamma_{r r}$ is the radial strain, $\dot{\gamma}_{r r}$ is the strain rate, $\mu$ is the viscosity, $u$ is the velocity, $u=\left(R^{2} / r^{2}\right) \dot{R}$ and $\dot{\gamma}_{r r}=\partial u / \partial r, G$ is the shear modulus. In the near field near the bubble surface, $\gamma_{r r}=\left(2 / 3 r^{3}\right)\left(R_{0}^{3}-\right.$ $\left.R^{3}\right), \gamma_{r r}=-\left(2 R^{2} / r^{3}\right) \dot{R}$, the shear stress integral is:

$$
3 \int_{R}^{\infty} \frac{\tau_{r r}}{r} d r=\frac{4 G}{3 R^{3}}\left(R_{0}^{3}-R^{3}\right)-\frac{4 \mu \dot{R}}{R}
$$

In summary, $P$ can be expanded as follows:

$$
P=p_{g}-p_{0}-\frac{2 \sigma}{R}+p_{a}-\left[\frac{4 G}{3 R^{3}}\left(R^{3}-R_{0}^{3}\right)+\frac{4 \mu \dot{R}}{R}\right]
$$

and

$$
\begin{aligned}
& \frac{d P}{d t}=\frac{d}{d t}\left(p_{g}-p_{0}-\frac{2 \sigma}{R}+p_{a}\right)+\frac{d}{d t}\left(3 \int_{R}^{\infty} \frac{\tau_{r r}}{r} d r\right) \\
& =\frac{d p_{g}}{d t}+\frac{2 \sigma \dot{R}}{R^{2}}+\frac{d p_{a}}{d t}-4 G \frac{R_{0}^{3} \dot{R}}{R^{4}}-4 \mu\left(-\frac{R^{2}}{R^{2}}+\frac{\ddot{R}}{R}\right)
\end{aligned}
$$

Combining Equation (3) and Equation (7), we can get:

$$
\frac{d P}{d t}=\left(\frac{2 \sigma}{R}-3 \kappa p_{g}\right) \frac{\dot{R}}{R}+\frac{d p_{a}}{d t}-4 G \frac{R_{0}^{3} \dot{R}}{R^{4}}+4 \mu\left(\frac{\dot{R^{2}}}{R^{2}}-\frac{\ddot{R}}{R}\right)
$$

For multifrequency ultrasound irradiation, the excitation signal can be expressed as follows:

$$
p_{a}=p_{1} \sin \left(2 \pi f_{1} t\right)+\phi p_{2} \sin \left(2 \pi f_{2} t+\alpha\right)+\varphi p_{3} \sin \left(2 \pi f_{3} t+\beta\right)
$$


where $f_{1}, f_{2}$, and $f_{3}$ are the frequency of excitation signal, $p_{1}, p_{2}$, and $p_{3}$ are the amplitude of sound pressure, $0 \leq \varphi \leq 1,0 \leq \phi \leq 1$. $\alpha$ and $\beta$ are the phase angle of the excitation signal, $0 \leq \alpha \leq 2 \pi$, $0 \leq \beta \leq 2 \pi$. Equations (7), (9) and (10) are substituted into Equation (1) to extract the equation with $\ddot{R}$, and then the ordinary differential equation solver (ode45) [24] in MATLAB (R2018b) software is used to solve it to obtain the bubble radius $R$. In this paper, the initial radius of bubble $R_{0}$ varies from $0.1 \mu \mathrm{m}$ to $10 \mu \mathrm{m}$, and the initial interface velocity between the bubble and the surrounding medium is zero $(\dot{R}=0)$. Finally, the bubble radius $R$ and Equation (3) and (10) are combined into Equation (7), and take $R_{\max }=2 R_{0}$ as the condition of transient cavitation [25], and the transient cavitation threshold in the medium can be solved.

(a) When $\phi=\varphi=0$, it is single-frequency $\left(f_{1}\right)$ excitation, $p_{1}=p_{0}$.

$$
p_{a}=p_{0} \sin \left(2 \pi f_{1} t\right)
$$

(b) When $\phi=1, \varphi=0, \alpha=0$, it is dual-frequency $\left(f_{1} \neq f_{2}\right)$ excitation, $p_{1}=p_{2}=\frac{1}{\sqrt{2}} p_{0}$.

$$
p_{a}=\frac{1}{\sqrt{2}} p_{0} \sin \left(2 \pi f_{1} t\right)+\frac{1}{\sqrt{2}} p_{0} \sin \left(2 \pi f_{2} t\right)
$$

(c) When $\phi=\varphi=1, \alpha=\beta=0$, it is triple-frequency $\left(f_{1} \neq f_{2} \neq f_{3}\right)$ excitation, $p_{1}=p_{2}=p_{3}=\frac{1}{\sqrt{3}} p_{0}$.

$$
p_{a}=\frac{1}{\sqrt{3}} p_{0} \sin \left(2 \pi f_{1} t\right)+\frac{1}{\sqrt{3}} p_{0} \sin \left(2 \pi f_{2} t\right)+\frac{1}{\sqrt{3}} p_{0} \sin \left(2 \pi f_{3} t\right)
$$

The above factors $\sqrt{2}$ and $\sqrt{3}$ are used to ensure that the ultrasound power of dual-frequency, triple-frequency, and single-frequency excitation is the same. The environmental pressure is $p_{0}=1.01 \times 10^{5} \mathrm{~Pa}$ in the simulation, the polytropic index represents the change of state parameters in the process of polytropic compression of gas, and it is usually taken as $\kappa=1.0 \sim 1.4$ [26]. If there is no special description in the following paper, the irradiation mode is single-frequency ultrasound, dual-frequency ultrasound, and triple-frequency ultrasound, and $\kappa$ is set to be 1.4. The transient cavitation threshold in water, blood, brain, and liver is studied by simulation, and the physical parameters of these four media are shown in Table 1.

Table 1. Physical parameters of media [27].

\begin{tabular}{cccccc}
\hline Medium & $\begin{array}{c}\text { Speed/c } \\
(\mathbf{m} / \mathbf{s})\end{array}$ & $\begin{array}{c}\text { Density/ } \boldsymbol{\rho} \\
\left(\mathbf{g} / \mathbf{m}^{\mathbf{3}}\right)\end{array}$ & $\begin{array}{c}\text { Surface Tension/ } \sigma \\
(\mathbf{m N} / \mathbf{m})\end{array}$ & $\begin{array}{c}\text { Viscosity/ } \boldsymbol{\mu} \\
(\mathbf{m P a . s})\end{array}$ & $\begin{array}{c}\text { Shear Modulus/G } \\
(\mathbf{M P a})\end{array}$ \\
\hline Water & 1500 & 1000 & 68 & 1 & 0 \\
Blood & 1570 & 1050 & 56 & 5 & 0 \\
Brain & 1540 & 1050 & 56 & 9 & 0.012 \\
Liver & 1549 & 1100 & 56 & 9 & 0.04 \\
\hline
\end{tabular}

\section{Simulation Results}

\subsection{Influence of Multifrequency Ultrasonic Combination on Transient Cavitation Threshold}

Figure 1 shows the relationship between the corresponding transient cavitation threshold and the initial bubble radius in water, blood, brain, and liver under different triple-frequency combined ultrasound irradiation. It can be seen from Figure $1 \mathrm{a}-\mathrm{d}$ that the transient cavitation threshold is different under different triple-frequency combined ultrasound irradiation. The transient cavitation threshold decreases first and then increases with the increase of the initial bubble radius. In addition, a "groove" appears in Figure 1, and this indicates that the ultrasonic frequency is close to the resonant frequency of the bubble or several times of the resonant frequency of the bubble. The resonant frequency of the bubble in fluid [28] and tissue [29,30] can be described by the following equations, respectively: 


$$
\begin{gathered}
f_{l}=\frac{1}{2 \pi R_{0} \sqrt{\rho}} \sqrt{3 \kappa\left(p_{0}-p_{g}+\frac{2 \sigma}{R_{0}}\right)-\frac{2 \sigma}{R_{0}}-\frac{4 \mu^{2}}{\rho R_{0}^{2}}} \\
f_{t}=\frac{1}{2 \pi R_{0} \sqrt{\rho}} \sqrt{3 \kappa\left(p_{0}-p_{g}\right)+(3 \kappa-1) \frac{2 \sigma}{R_{0}}-\frac{8 \mu^{2}}{\rho R_{0}^{2}}+4 G}
\end{gathered}
$$

(a)

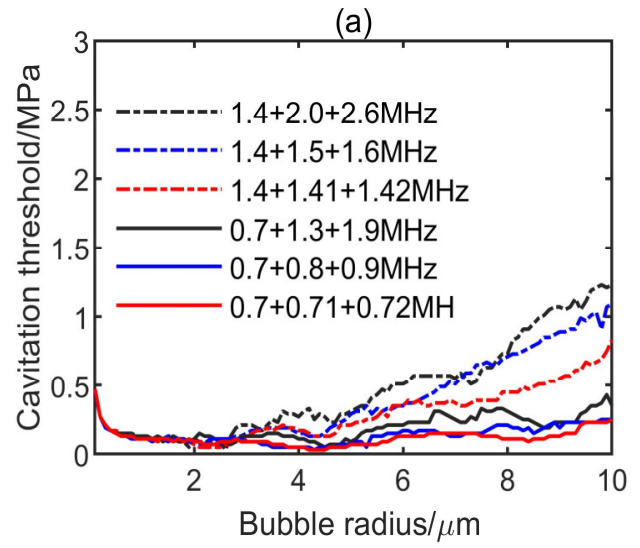

(c)

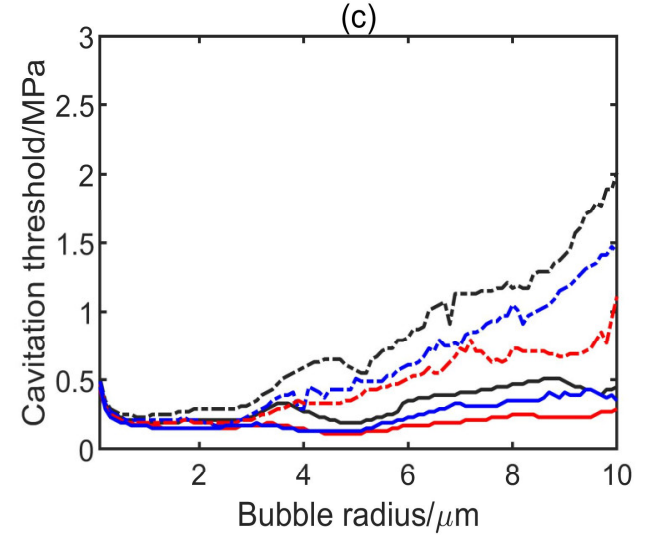

(b)

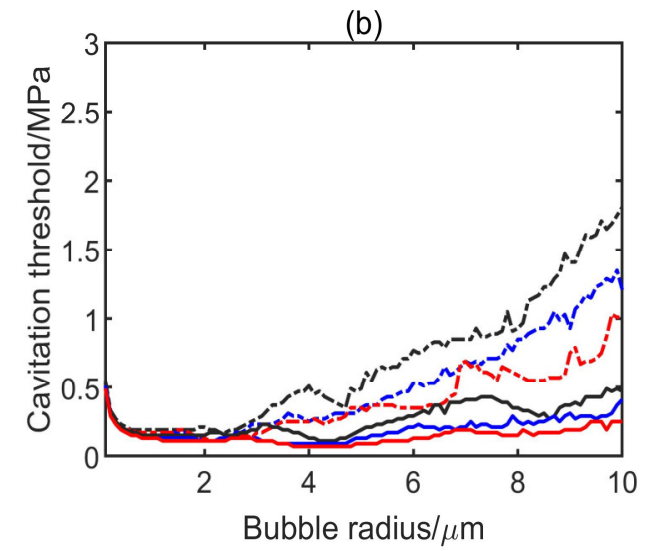

(d)

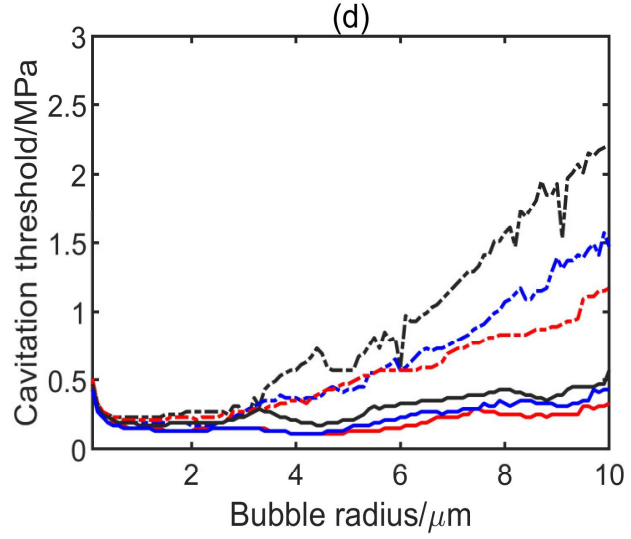

Figure 1. Transient cavitation threshold corresponding to different triple-frequency combinations in different media: (a) water; (b) blood; (c) brain; (d) liver.

Because the transient cavitation threshold is closely related to the initial bubble size, the effectiveness of this study mainly depends on the existence of stable small bubble nuclei. It has been reported that the radius of cavitation nuclei in water was about $1 \mu \mathrm{m}$ or less, and distribution density decreased exponentially with the radius of the nuclei [31,32]. However, the nuclei radius in vivo was estimated to be less than $0.3 \mu \mathrm{m}[33,34]$. Therefore, the bubble size in the range of $0.1 \mu \mathrm{m}$ to $10 \mu \mathrm{m}$ used here is close to the actual situation. Taking the triple-frequency combination of $1.4+2.0+$ $2.6 \mathrm{MHz}$ in Figure 1 as an example, when the initial bubble radius is $10 \mu \mathrm{m}$, the corresponding transient cavitation threshold from large to small in the media are: liver, brain, blood, and water. Therefore, for the high-frequency combination, the higher the viscosity and shear modulus of the medium, the higher the change trend of the transient cavitation threshold under the same and larger initial bubble radius.

For any combination of triple frequency in Figure $1 \mathrm{a}-\mathrm{d}$, the transient cavitation threshold of the combination of triple frequency with higher frequency (such as $1.4+1.41+1.42 \mathrm{MHz}$ ) is higher than that of the combination of triple frequency with lower frequency (such as $0.7+0.71+0.72 \mathrm{MHz}$ ) and when the frequency difference is the same, the higher combination frequency and viscosity of triple frequency will increase the transient cavitation threshold. The higher frequency results in an increase of the cavitation threshold. The inertia of large expansion ratios leads to the suppression of bubble collapse in the positive half cycle, and then the bubble continues to grow in the following negative 
half cycle, resulting in the higher transient cavitation threshold [35]. In Figure 1, the triple-frequency combination with high frequency corresponding to the transient cavitation threshold from low to high are $1.4+1.41+1.42 \mathrm{MHz}, 1.4+1.5+1.6 \mathrm{MHz}$, and $1.4+2.0+2.6 \mathrm{MHz}$, but the triple-frequency combination with low frequency corresponding to the transient cavitation threshold from low to high are $0.7+0.71+0.72 \mathrm{MHz}, 0.7+0.8+0.9 \mathrm{MHz}$, and $0.7+1.3+1.9 \mathrm{MHz}$. When the lowest frequency of the triple-frequency combination is the same, the larger the frequency difference is, the higher the corresponding transient cavitation threshold is. When the initial bubble radius is small, the influence of frequency difference on the transient cavitation threshold of different triple-frequency combinations is not obvious. With the increase of initial bubble radius, the influence of frequency difference on the transient cavitation threshold of the high-frequency triple-frequency combination is more obvious, but the influence on the transient cavitation threshold of the low-frequency triple-frequency combination is not obvious.

Figure 2 shows the relationship between the transient cavitation threshold and the initial bubble radius in water, blood, brain, and liver under the combination of single-frequency $(2.6 \mathrm{MHz})$, dual-frequency $(2.6+2.0 \mathrm{MHz})$, and triple-frequency $(2.6+2.0+1.4 \mathrm{MHz})$. For these four different media, under the same initial bubble radius, the transient cavitation threshold of the dual-frequency combination is lower than that of the single-frequency ultrasound, and the transient cavitation threshold of the triple-frequency combination is lower than that of the dual-frequency combination. With the increase of the initial bubble radius, the difference between the transient cavitation threshold of the dual-frequency and triple-frequency combination and that of the single-frequency ultrasound gradually increases, but the difference between the transient cavitation threshold of the dual-frequency and triple-frequency combination is not significant. The results show that when the highest frequency of the multifrequency combination is the same as that of the single-frequency ultrasound, the transient cavitation threshold of the multifrequency combination is lower than that of the single-frequency ultrasound. Compared with the single-frequency ultrasound, the smaller the initial bubble radius, the smaller the effect of the multifrequency combination on reducing the transient cavitation threshold. The larger the initial bubble radius is, the better the effect of the multifrequency combination to reduce the transient cavitation threshold is. It is generally believed that if the ultrasound with lower frequency is introduced into the multifrequency combination, the transient cavitation threshold will be reduced $[10,16,27,36]$. The simulation results are consistent with the experimental results of Feng et al. [15], who used multifrequency irradiation to enhance cavitation: compared with the single frequency ultrasound, the dual-frequency combination can significantly enhance the cavitation effect, while the triple-frequency combination can further enhance the cavitation effect.

Figure 3 shows the relationship between the transient cavitation threshold and the initial bubble radius in water, blood, brain, and liver under the combination of single-frequency (1.4 MHz), dual-frequency $(1.4+2.0 \mathrm{MHz})$, and triple-frequency $(1.4+2.0+2.6 \mathrm{MHz})$. Compared with Figure 2, the single-frequency in Figure 3 is reduced, and the dual-frequency and triple-frequency are combined in the way of increasing frequency. For these four different media, under the same initial bubble radius, the transient cavitation threshold of the dual-frequency combination is higher than that of the single-frequency ultrasound, and the transient cavitation threshold of the triple-frequency combination is higher than that of the dual-frequency combination. The simulation results in Figure 3 are just opposite to those in Figure 2. When the lowest frequency of the multifrequency combination is the same as that of single-frequency ultrasound, the transient cavitation threshold of the multifrequency combination is higher than that of the single-frequency ultrasound. The simulation results in Figure 3 show that the transient cavitation threshold change from the single-frequency ultrasound to the dual-frequency combination is stronger than that from the dual-frequency combination to the triple-frequency combination, which is consistent with the experimental results of Suo [13] and other researchers $[37,38]$ who used multifrequency excitation to enhance cavitation. 
(a)

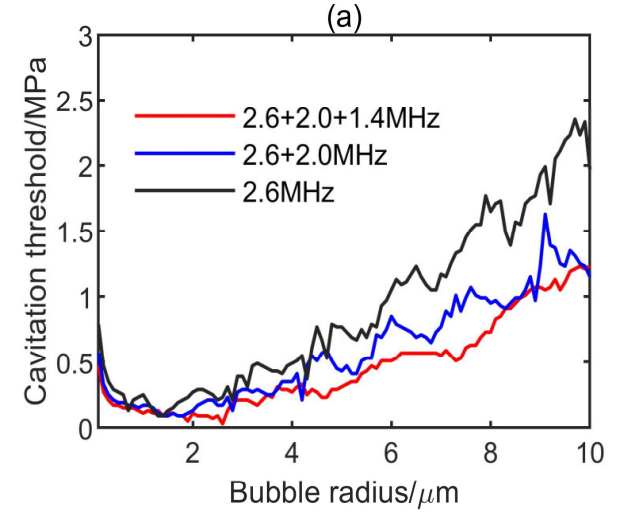

(c)

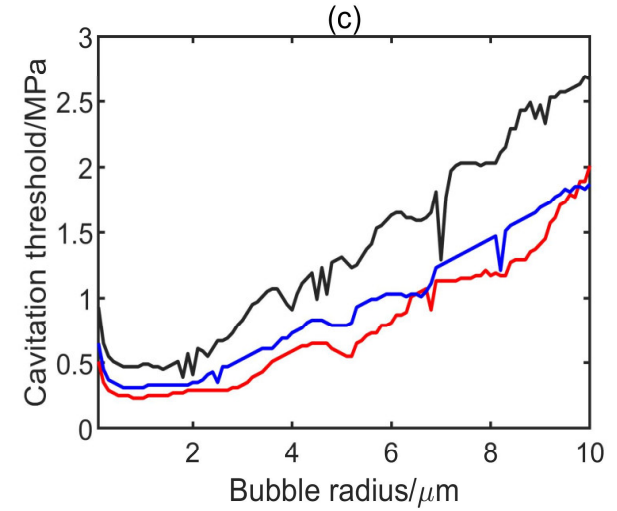

(b)

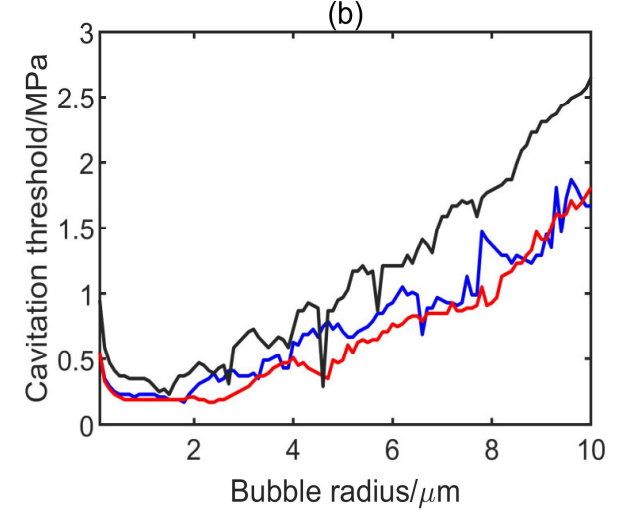

(d)

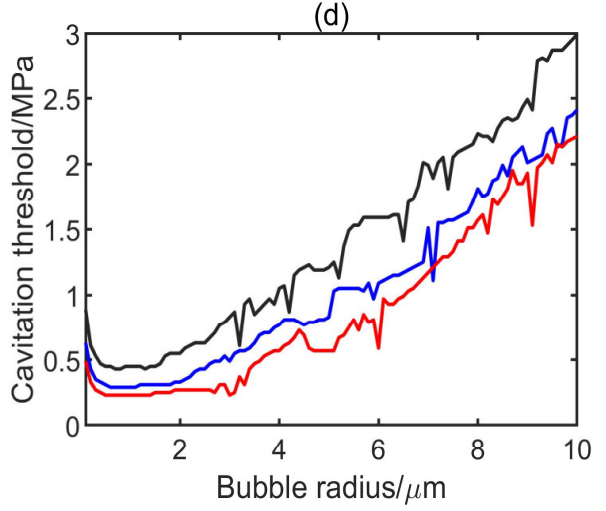

Figure 2. Transient cavitation threshold of single-frequency $(2.6 \mathrm{MHz})$, dual-frequency $(2.6+2.0 \mathrm{MHz})$, and triple-frequency $(2.6+2.0+1.4 \mathrm{MHz})$ in different media: (a) water; (b) blood; (c) brain; (d) liver.

(a)

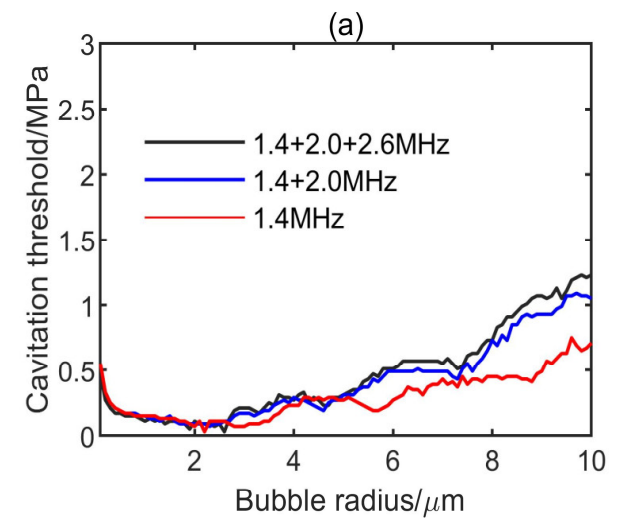

(c)

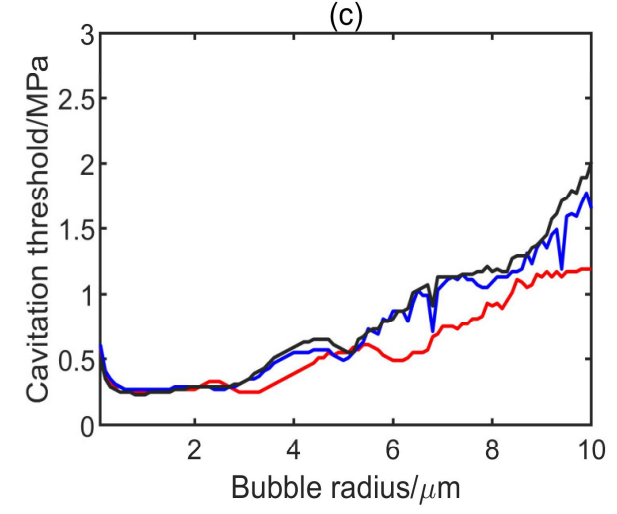

(b)

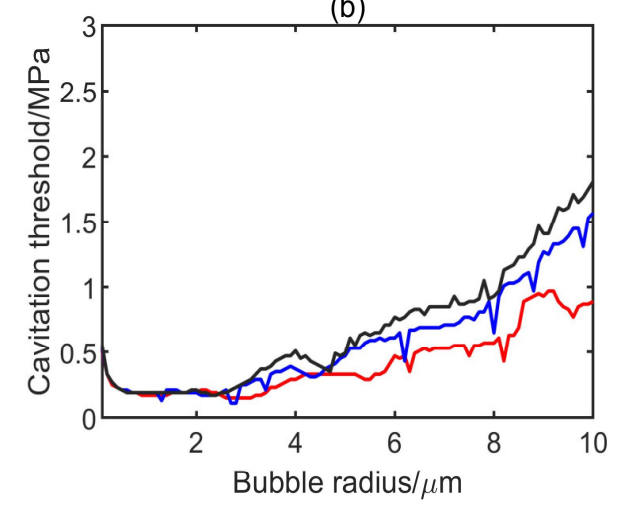

(d)

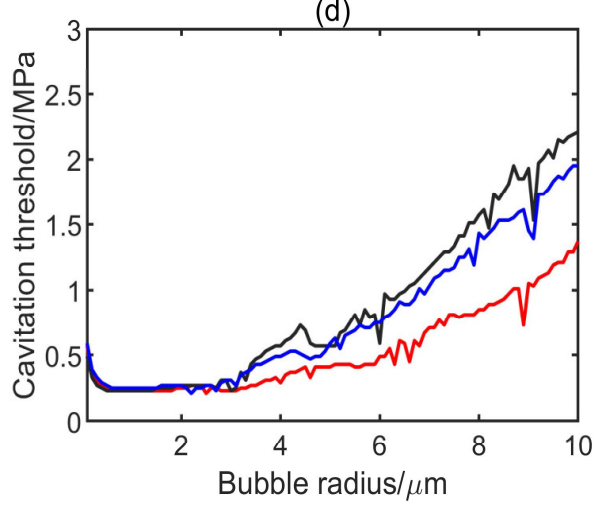

Figure 3. Transient cavitation threshold of single-frequency $(1.4 \mathrm{MHz})$, dual-frequency $(1.4+2.0 \mathrm{MHz})$, and triple-frequency $(1.4+2.0+2.6 \mathrm{MHz})$ in different media: (a) water; (b) blood; (c) brain; (d) liver. 


\subsection{Effect of Peak Negative Pressure and Its Duration on Transient Cavitation Threshold}

For a single spherical bubble in the medium, the dynamic response of the bubble can be evaluated by the initial bubble radius, the peak negative pressure of the ultrasound, and its duration. When the maximum radius of the bubble is $R_{\max }=2 R_{0}$, the corresponding transient cavitation threshold is closely related to the peak negative pressure and its duration $[39,40]$. Taking Figure $2 \mathrm{~d}$ as an example, Figure 4 theoretically analyzes the reason for the difference of transient cavitation threshold of liver under the combined irradiation of $2.6 \mathrm{MHz}, 2.6+2.0 \mathrm{MHz}$, and $2.6+2.0+1.4 \mathrm{MHz}$.

(a)

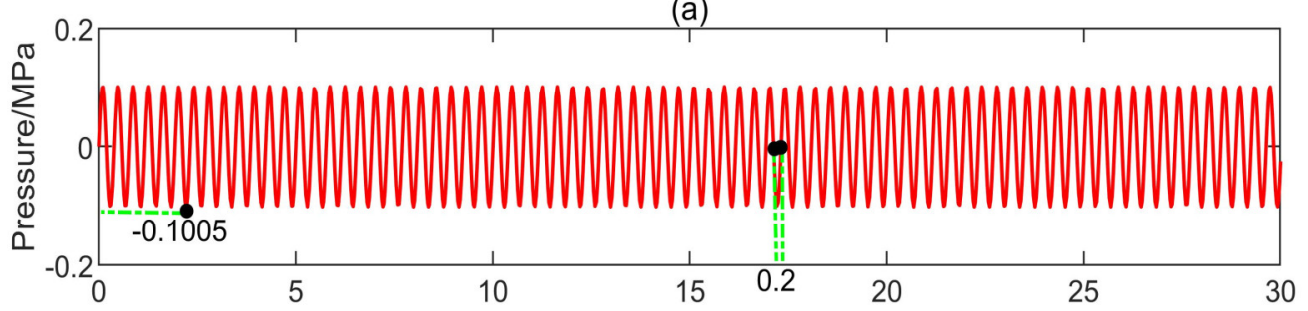

(b)

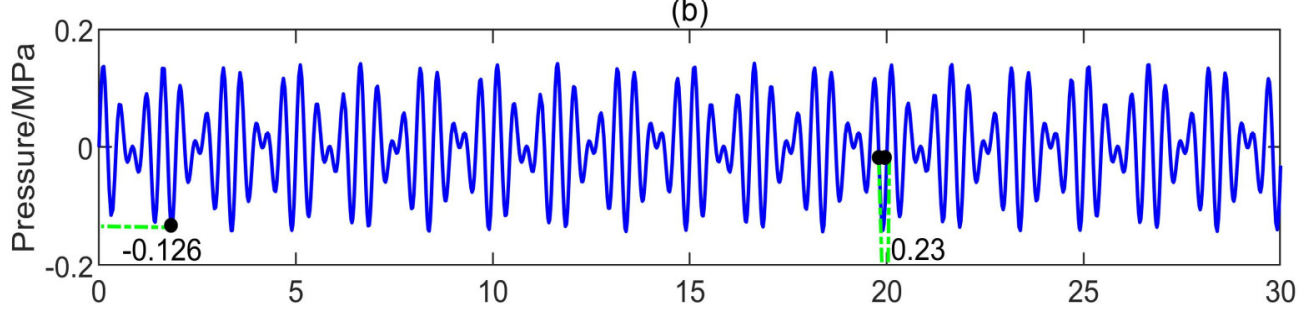

(c)

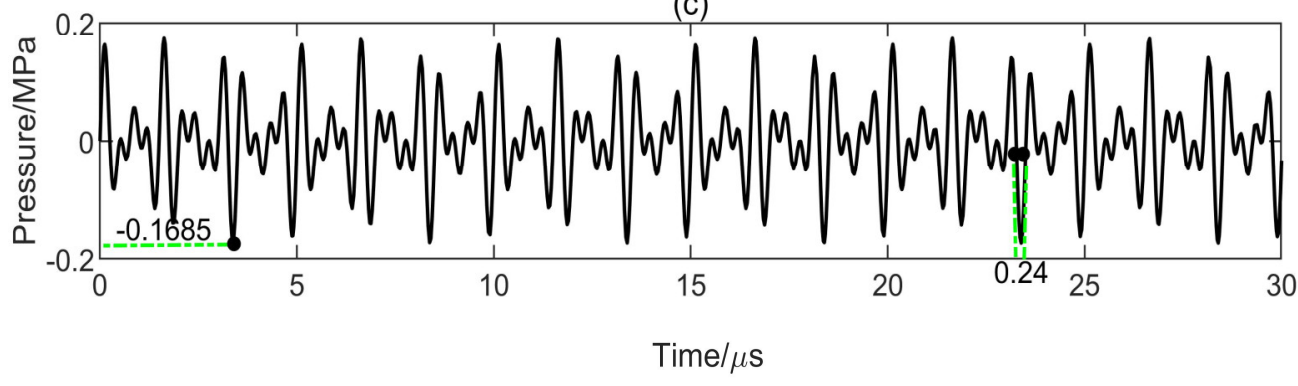

Figure 4. Pressure waveforms of different frequency combinations in liver: (a) $2.6 \mathrm{MHz}$; (b) $2.6+2.0 \mathrm{MHz}$; (c) $2.6+2.0+1.4 \mathrm{MHz}$.

The initial bubble radius is $5 \mu \mathrm{m}$ and the initial pressure is $0.101 \mathrm{MPa}$ under the combination of three different frequencies of ultrasonic irradiation in Figure 4. It can be found from Figure 4 that from the single-frequency ultrasound to the triple-frequency combination, their peak negative pressure gradually increases, and the peak negative pressure values of the single-frequency $(2.6 \mathrm{MHz})$, dual-frequency $(2.6+2.0 \mathrm{MHz})$, and triple-frequency $(2.6+2.0+1.4 \mathrm{MHz})$ combinations are $-0.1005 \mathrm{MPa},-126 \mathrm{MPa}$, and $-0.1685 \mathrm{MPa}$, respectively. In general, under the same ultrasound power, the peak negative pressure corresponding to the triple-frequency and dual-frequency combinations is greater than that corresponding to the single-frequency [13]. At the same time, the peak negative pressure duration of the single-frequency, dual-frequency, and triple-frequency combinations are $0.2 \mu \mathrm{s}, 0.23 \mu \mathrm{s}$, and $0.24 \mu \mathrm{s}$, respectively. It can be seen from Figure 4 that the duration of peak negative pressure of the three different combined frequencies of ultrasound is relatively small, but the difference of peak negative pressure is relatively large. The peak negative pressure of the triple-frequency combination is the largest while that of the single-frequency ultrasound is the smallest. The increase of peak negative pressure reduces the cavitation threshold of the medium, and the probability and intensity of cavitation are also greater [41]. It is concluded that when the peak negative pressure of the triple-frequency combination is the largest and the peak negative pressure of the single-frequency ultrasound is the 
smallest, the transient cavitation threshold of the triple-frequency combination is the lowest, and the transient cavitation threshold of the single-frequency ultrasound is the highest.

In the same way, taking Figure $3 \mathrm{~d}$ as an example, Figure 5 theoretically analyzes the reason for the difference of transient cavitation threshold of liver under the combined irradiation of $1.4 \mathrm{MHz}$, $1.4+2.0 \mathrm{MHz}$, and $1.4+2.0+2.6 \mathrm{MHz}$.

(a)

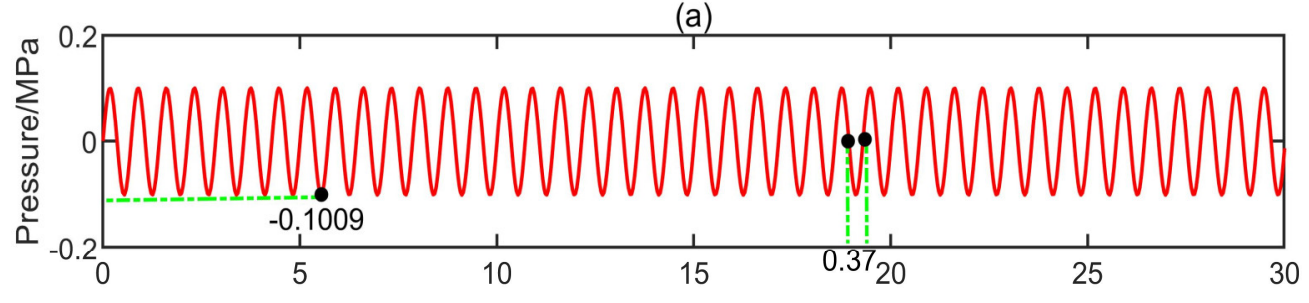

(b)

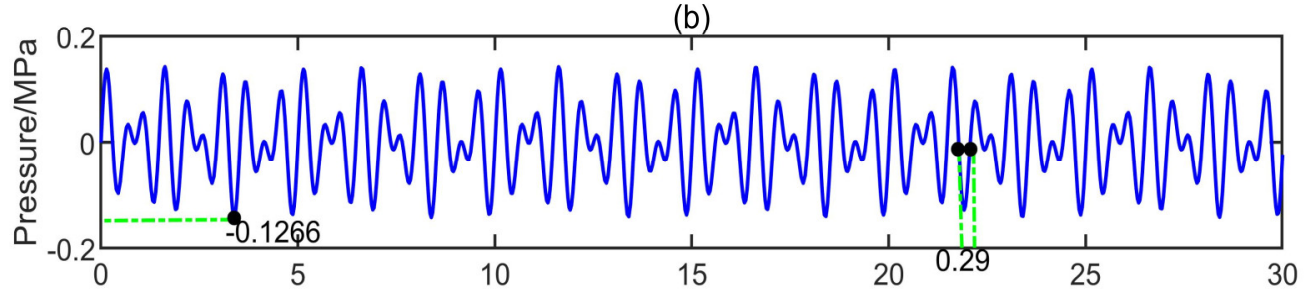

(c)

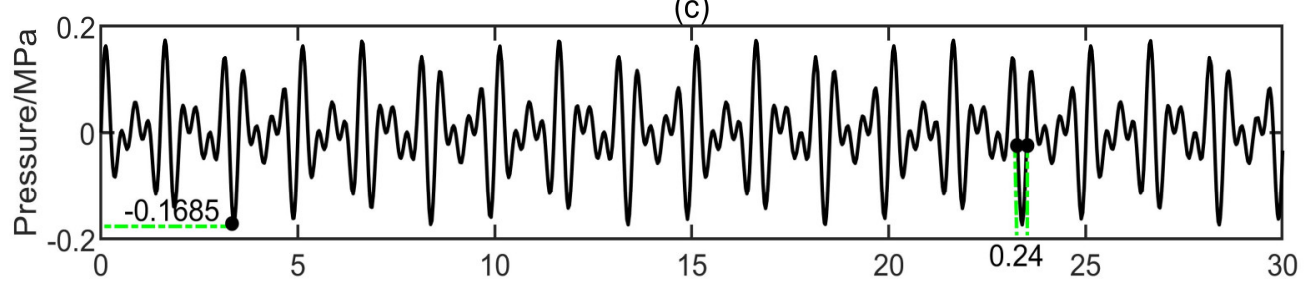

$\mathrm{Time} / \mu \mathrm{s}$

Figure 5. Pressure waveforms of different frequency combinations in liver: (a) $1.4 \mathrm{MHz}$; (b) $1.4+2.0 \mathrm{MHz}$; (c) $1.4+2.0+2.6 \mathrm{MHz}$.

In Figure 5, the initial bubble radius is $5 \mu \mathrm{m}$ and the initial pressure is $0.101 \mathrm{MPa}$ under three different frequencies combined ultrasonic irradiation. From Figure 5, it can be found that the peak negative pressures of the single-frequency $(1.4 \mathrm{MHz})$, dual-frequency $(1.4+2.0 \mathrm{MHz})$, and triple-frequency $(1.4+2.0+2.6 \mathrm{MHz})$ combined ultrasound are $-0.1009 \mathrm{MPa},-0.1266 \mathrm{MPa}$, and $-0.1685 \mathrm{MPa}$, respectively. At the same time, the peak negative pressure durations of the single-frequency, dual-frequency, and triple-frequency ultrasound are $0.37 \mu \mathrm{s}, 0.29 \mu \mathrm{s}$, and $0.24 \mu \mathrm{s}$, respectively. Under the ultrasonic irradiation of three different combined frequencies, the peak negative pressure of the corresponding ultrasound is $\sim 0.1 \mathrm{MPa}$, and the difference is not very large. However, their peak negative pressure duration is quite different. The longer the peak negative pressure duration is in a certain time range, the stronger the cavitation intensity is [42]. This is also the reason why the transient cavitation threshold of the dual-frequency combination is higher than that of the single-frequency ultrasound, and the transient cavitation threshold of the triple-frequency combination is higher than that of the dual-frequency combination.

\subsection{Effect of Phase Angle Difference on Transient Cavitation Threshold}

The phase angle difference of the ultrasonic excitation signal has an influence on the distribution of sound pressure and the occurrence of bubble chaotic oscillation [43], and it also has an influence on the 
transient cavitation threshold in the medium. In order to study the influence of different phase angle differences of the multifrequency excitation signal on the transient cavitation threshold, taking the three different kinds of phase angle combinations of triple-frequency $(2.6+2.0+1.4 \mathrm{MHz})$ excitation as an example, the phase angle of $2.6 \mathrm{MHz}$ excitation is set to 0 , and that of $2.0 \mathrm{MHz}$ and $1.4 \mathrm{MHz}$ excitation are $\alpha$ and $\beta$, respectively, and the phase angles of $2.0 \mathrm{MHz}$ and $1.4 \mathrm{MHz}$ excitation are $\alpha=\beta=0$, $\alpha=2 \pi / 3, \beta=\pi$ and $\alpha=2 \pi / 3, \beta=4 \pi / 3$, respectively. The phase angle difference between them is gradually increasing, and the phase angle differences are $0, \pi / 2$, and $2 \pi / 3$, respectively. Figure 6 shows the relationship between the transient cavitation threshold and the initial bubble radius under triple-frequency excitation with different phase angle differences in water, blood, brain, and liver.

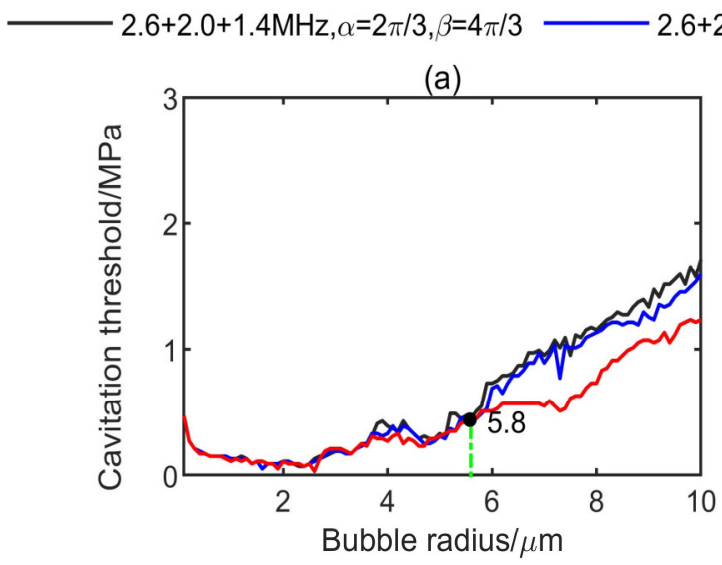

(c)
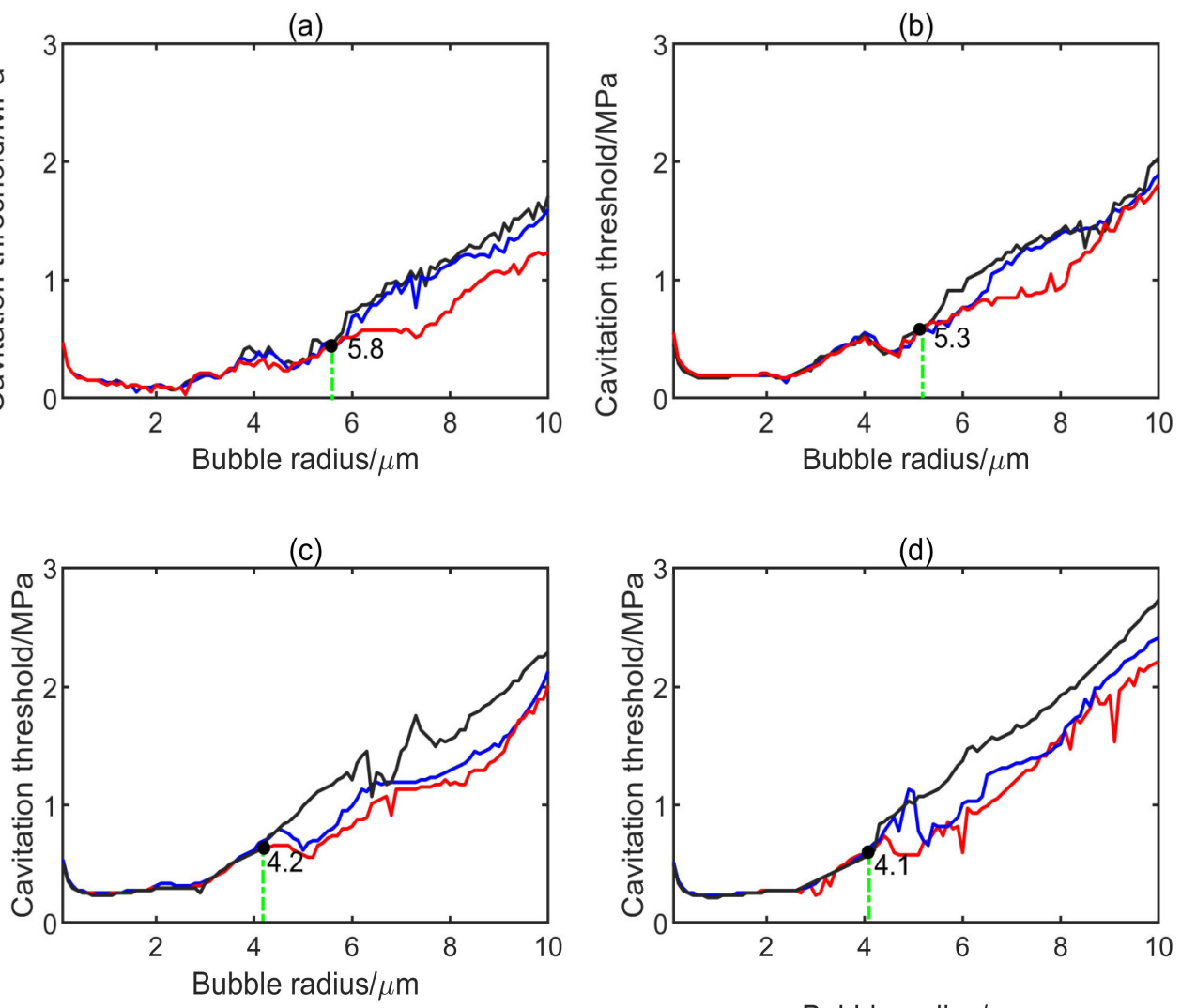

(d)

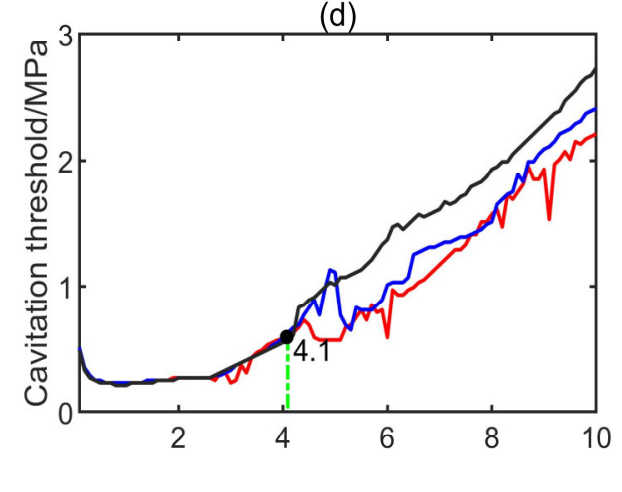

Bubble radius $/ \mu \mathrm{m}$

Figure 6. Transient cavitation threshold of triple-frequency $(2.6+2.0+1.4 \mathrm{MHz})$ excitation with different phase angle differences in different media: (a) water; (b) blood; (c) brain; (d) liver.

It can be seen from Figure 6 that when the initial bubble radius in water, blood, brain, and liver is small, the transient cavitation threshold excited by triple-frequency $(2.6+2.0+1.4 \mathrm{MHz})$ is almost not affected by the change of phase angle difference at first, which may be caused by the nonlinear decrease of bubble oscillation [44]. The amplitude of transient cavitation threshold in brain and liver fluctuates less than that in water and blood. When the bubble radius is larger, the corresponding transient cavitation threshold is more sensitive to the phase angle difference of the excitation, which is more obvious in water and blood. Specifically, when the initial bubble radius in water, blood, brain, and liver is greater than $5.8 \mu \mathrm{m}, 5.3 \mu \mathrm{m}, 4.2 \mu \mathrm{m}$, and $4.1 \mu \mathrm{m}$, respectively, the cavitation threshold of different phase angle differences is significantly different. Under the same larger initial bubble radius, when the phase angle is $\alpha=\beta=0$, the phase angle difference of the triple-frequency excitation is 0 , and the transient cavitation threshold is the lowest compared with other phase angle differences; when the phase angle is $\alpha=2 \pi / 3, \beta=4 \pi / 3$, the phase angle difference of the triple-frequency excitation is $2 \pi / 3$, and the transient cavitation threshold of these four media is at the highest value compared 
with other phase angle differences. With the increase of the phase angle difference of triple-frequency excitation, the corresponding transient cavitation threshold of the triple-frequency excitation in the medium increases gradually. Therefore, the phase angle difference of the multifrequency excitation has an impact on the transient cavitation threshold in the medium. In the actual HIFU treatment process, the lower transient cavitation threshold can be obtained by selecting the appropriate phase angle difference of the multifrequency excitation.

\subsection{Effect of Polytropic Index on Transient Cavitation Threshold}

The polytropic index controls the thermal dynamic behavior of the gas in the bubble. The polytropic index $k=1.4$ corresponds to the adiabatic process of the bubble, while $k=1.0$ corresponds to the isothermal process. The relevant research shows that the adiabatic process underestimates the maximum radius of the bubble, while the isothermal process overestimates the maximum radius of the bubble $[26,45]$. Figure 7 takes the liver as an example to analyze the influence of the polytropic index on the transient cavitation threshold under different frequency combinations by changing the value of the polytropic index. Figure 7 shows the relationship between the transient cavitation threshold and the initial bubble radius under the combinations of single-frequency $(2.6 \mathrm{MHz})$, dual-frequency $(2.6+2.0 \mathrm{MHz})$, and triple-frequency $(2.6+2.0+1.4 \mathrm{MHz})$.
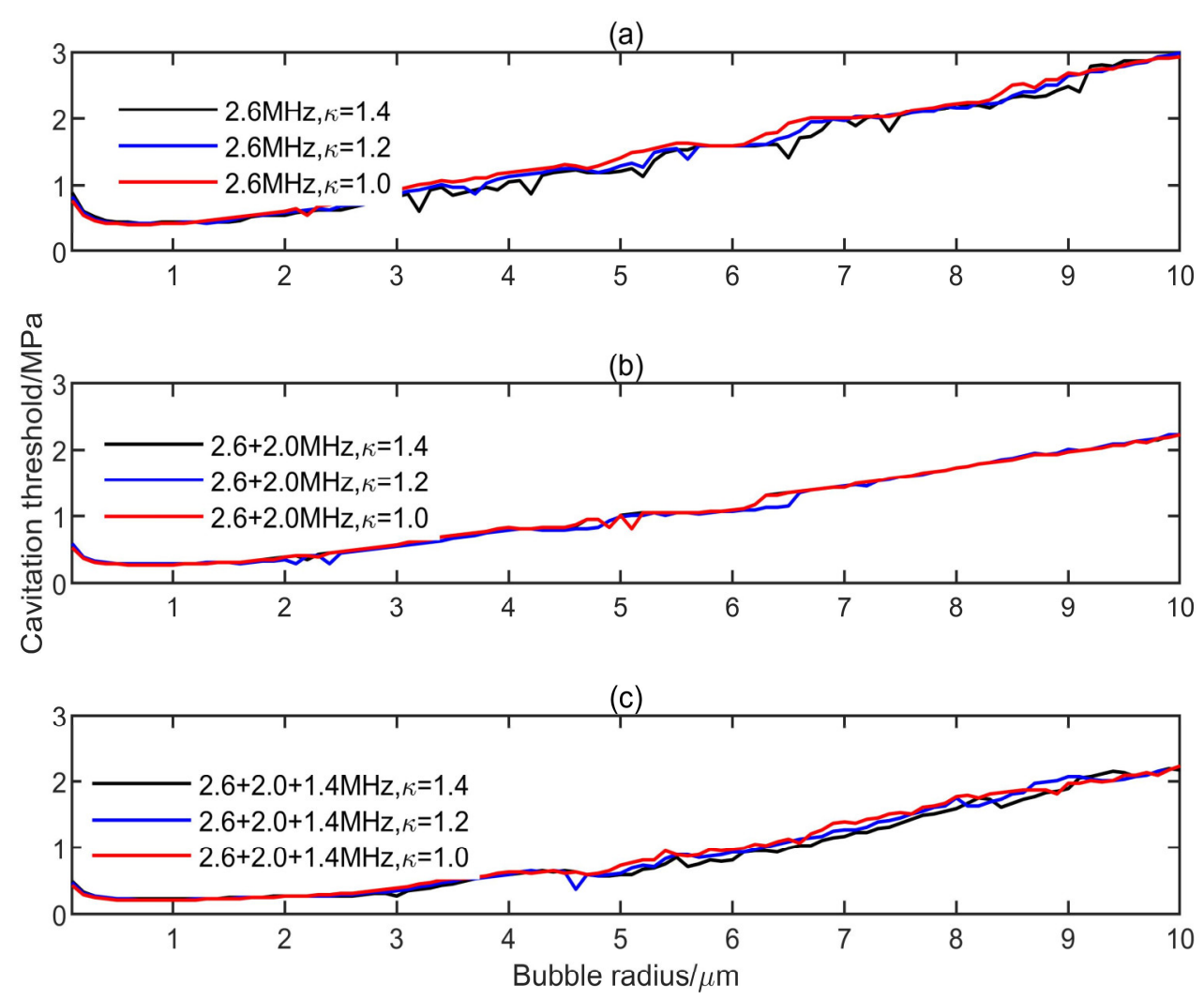

Figure 7. The influence of polytropic index on transient cavitation threshold under three different frequency combinations: (a) $2.6 \mathrm{MHz}$; (b) $2.6+2.0 \mathrm{MHz}$; (c) $2.6+2.0+1.4 \mathrm{MHz}$.

It can be found from Figure 7 that under the same irradiation conditions, the change of polytropic index has little effect on the transient cavitation threshold of multifrequency combinations in the medium, whether it is a single-frequency ultrasound, dual-frequency combination, or triplefrequency combination. 


\section{Conclusions}

In this paper, the Yang-Church model is used to simulate the nonlinear vibration of bubbles in thermally viscous media. The effects of multifrequency ultrasonic combination mode, peak negative pressure and its duration, phase angle difference, and polytropic index on the transient cavitation threshold in different media are analyzed. The simulation results show that:

(1) On the premise of the same frequency difference and initial bubble radius, the transient cavitation threshold of the triple-frequency combination with higher frequency is higher than that of the triple-frequency combination with lower frequency. With the increase of the initial bubble radius, the threshold difference between the single-frequency ultrasound and the multifrequency combination increases, and the influence of the frequency difference on the transient cavitation threshold of the triple-frequency combination with higher frequency is more obvious.

(2) When the lowest frequency of triple frequencies is the same, the larger the frequency difference is, the higher the corresponding transient cavitation threshold is. When the bubble radius is small, the frequency difference has little effect on the transient cavitation threshold of the triple-frequency combination.

(3) The level of transient cavitation threshold is related to the peak negative pressure and duration of ultrasound. Compared with the single-frequency ultrasound, the introduction of a low-frequency component in the multifrequency combination can significantly reduce the transient cavitation threshold in the medium. However, the introduction of a high-frequency component in the multifrequency combination can significantly increase the transient cavitation threshold in the medium.

(4) When the phase angle difference of the multifrequency excitation is zero, the corresponding transient cavitation threshold in the medium is the lowest. The change of the polytropic index has little effect on the transient cavitation threshold of different frequency combinations.

The results of this study can provide guidance for multifrequency ultrasound in frequency combination, frequency difference, and phase angle selection, so as to better reduce the transient cavitation threshold in the medium, and provide reference for the design of tissue ablation treatment schemes in clinical application.

Author Contributions: H.D. conceived and designed the research idea and the framework; H.D. and X.Z. performed the simulations; H.D. and X.Z. wrote the paper; S.Q., H.D., and X.Z. analyzed the data, S.Q. modified the paper. All authors have read and agreed to the published version of the manuscript.

Funding: This research received no external funding.

Acknowledgments: This work was supported by National Natural Science Foundation of China (Grant No.11774088, 11474090), the Hunan Provincial Natural Science Foundation of China (Grant No.2018JJ3557), and the key Program of Changsha Normal University (Grant No. 2019XJZK12).

Conflicts of Interest: The authors declare no conflict of interest.

\section{References}

1. Holland, C.K.; Apfel, R.E. Thresholds for transient cavitation produced by pulsed ultrasound in a controlled nuclei environment. J. Acoust. Soc. Am. 1990, 88, 2059-2069. [CrossRef] [PubMed]

2. Helfield, B.; Black, J.J.; Qin, B.; Pacella, J.; Chen, X.; Villanueva, F.S. Fluid viscosity affects the fragmentation and inertial cavitation threshold of lipid-encapsulated microbubbles. Ultrasound Med. Biol. 2016, 42, $782-794$. [CrossRef]

3. Yang, Q.; Tang, P.; He, G.; Ge, S.; Liu, L.; Zhou, X. Hemocoagulase combined with microbubble-enhanced ultrasound cavitation for augmented ablation of microvasculature in rabbit VX2 liver tumors. Ultrasound Med. Biol. 2017, 43, 1658-1670. [CrossRef] [PubMed]

4. Thudium, M.; Bette, B.; Tonguc, T.; Ghaei, S.; Conrad, R.; Becher, M.U.; Mücke, M.; Luechters, G.; Strunk, S.; Marinova, M. Multidisciplinary management and outcome in pancreatic cancer patients treated with high-intensity focused ultrasound. Int. J. Hyperther. 2020, 37, 456-462. [CrossRef] [PubMed] 
5. Izadifar, Z.; Chapman, D.; Babyn, P. An Introduction to High Intensity Focused Ultrasound: Systematic Review on Principles, Devices, and Clinical Applications. J. Clin. Med. 2020, 9, 460. [CrossRef]

6. Guo, S.; Jiang, X.; Lin, W. Tissue ablation using multi-frequency focused ultrasound. In Proceedings of the IEEE International Ultrasonics Symposium, Orlando, FL, USA, 18-21 October 2011; pp. 2177-2180.

7. Law, S.K.B.; Zhou, Y. High-Intensity Focused Ultrasound Ablation by the Dual-Frequency Excitation. IEEE Trans. Ultrason. Ferroelectr. Freq. Control 2018, 66, 18-25.

8. Wang, Y.; Zhang, Z.; He, R.; Liu, D.; Mintah, B.K.; Dabbour, M.; Ma, H. Improvement in enzymolysis efficiency and changes in conformational attributes of corn gluten meal by dual-frequency slit ultrasonication action. Ultrason. Sonochem. 2020, 64, 105038. [CrossRef]

9. Ma, J.; Guo, S.; Wu, D.; Geng, X.; Jiang, X. Design, fabrication, and characterization of a single-aperture 1.5-MHz/3-MHz dual-frequency HIFU transducer. IEEE Trans. Ultrason. Ferroelectr. Freq. Control 2013, 60, 1519-1529. [CrossRef]

10. He, P.Z.; Shou, W.D.; Duan, S.M.; Xia, R.M. Dual-frequency high intensity focused ultrasound (HIFU) accelerating therapy. In Proceedings of the IEEE Engineering in Medicine and Biology 27th Annual Conference, Shanghai, China, 1-4 September 2005; pp. 213-216.

11. Gilles, B.; Béra, J.C.; Mestas, J.L.; Cathignol, D. Reduction of ultrasound inertial cavitation threshold using bifrequency excitation. Appl. Phys. Lett. 2006, 89, 94106. [CrossRef]

12. Saletes, I.; Gilles, B.; Auboiroux, V.; Bendridi, N.; Salomir, R.; Béra, J.C. In vitro demonstration of focused ultrasound thrombolysis using bifrequency excitation. Biomed. Res. Int. 2014, 1, 10.

13. Suo, D.; Guo, S.; Lin, W.; Jiang, X.; Jing, Y. Thrombolysis using multi-frequency high intensity focused ultrasound at MHz range: An in vitro study. Phys. Med. Biol. 2015, 60, 7403-7418. [CrossRef] [PubMed]

14. Sasaki, H.; Yasuda, J.; Takagi, R.; Miyashita, T.; Goto, K.; Yoshizawa, S.; Umemura, S.I. Highly efficient cavitation-enhanced heating with dual-frequency ultrasound exposure in high-intensity focused ultrasound treatment. Jpn. J. Appl. Phys. 2014, 53, 07KF11. [CrossRef]

15. Feng, R.; Zhao, Y.; Zhu, C.; Mason, T.J. Enhancement of ultrasonic cavitation yield by multi-frequency sonication. Ultrason. Sonochem. 2002, 9, 231-236. [CrossRef]

16. Guo, S.; Jing, Y.; Jiang, X. Temperature rise in tissue ablation using multi-frequency ultrasound. IEEE Trans. Ultrason. Ferroelectr. Freq. Control. 2013, 60, 1699-1707.

17. Wallace, N.; Dicker, S.; Lewin, P.; Wrenn, S.P. Inertial cavitation threshold of nested microbubbles. Ultrasonics 2015, 58, 67-74. [CrossRef]

18. Liu, R.; Xu, S.; Hu, H.; Huo, R.; Wang, S.; Wan, M. Wavelet-transform-based active imaging of cavitation bubbles in tissues induced by high intensity focused ultrasound. J. Acoust. Soc. Am. 2016, 140, 798-805. [CrossRef]

19. Yang, X.; Church, C.C. A model for the dynamics of gas bubbles in soft tissue. J. Acoust. Soc. Am. 2005, 118, 3595-3606. [CrossRef]

20. Fourest, T.; Deletombe, E.; Faucher, V.; Arrigoni, M.; Dupas, J.; Laurens, J.M. Comparison of Keller-Miksis model and finite element bubble dynamics simulations in a confined medium. Application to the Hydrodynamic Ram. Eur. J. Mech. B Fluid 2018, 68, 66-75. [CrossRef]

21. Man, V.H.; Li, M.S.; Derreumaux, P.; Nguyen, P.H. Rayleigh-Plesset equation of the bubble stable cavitation in water: A nonequilibrium all-atom molecular dynamics simulation study. J. Chem. Phys. 2018, 148, 094505. [CrossRef]

22. Vlaisavljevich, E.; Lin, K.W.; Warnez, M.T.; Singh, R.; Mancia, L.; Putnam, A.J.; Johnsen, E.; Cain, C.; Xu, Z. Effects of tissue stiffness, ultrasound frequency, and pressure on histotripsy-induced cavitation bubble behavior. Phys. Med. Biol. 2015, 60, 2271-2292. [CrossRef]

23. Suo, D.; Govind, B.; Zhang, S.; Jing, Y. Numerical investigation of the inertial cavitation threshold under multi-frequency ultrasound. Ultrason. Sonochem. 2018, 41, 419-426. [CrossRef] [PubMed]

24. Shampine, L.F.; Reichelt, M.W. The MATLAB ODE Suite. SIAM J. Sci. Comput. 1997, 18, 1-22. [CrossRef]

25. Wang, M.; Zhou, Y. Numerical investigation of the inertial cavitation threshold by dual-frequency excitation in the fluid and tissue. Ultrason. Sonochem. 2018, 42, 327-338. [CrossRef] [PubMed]

26. Maiga, M.A.; Coutier-Delgosha, O.; Buisine, D. Analysis of the critical pressure of cavitation bubbles. Meccanica 2018, 53, 787-801. [CrossRef] 
27. Church, C.C.; Labuda, C.; Nightingale, K. A Theoretical Study of Inertial Cavitation from Acoustic Radiation Force Impulse Imaging and Implications for the Mechanical Index1. Ultrasound Med. Biol. 2015, 41, 472-485. [CrossRef] [PubMed]

28. Lauterborn, W.; Kurz, T. Physics of bubble oscillations. Rep. Prog. Phys. 2010, 73, 106501. [CrossRef]

29. Gaudron, R.; Warnez, M.T.; Johnsen, E. Bubble dynamics in a viscoelastic medium with nonlinear elasticity. J. Fluid Mech. 2015, 766, 54-75. [CrossRef]

30. Khismatullin, D.B. Resonance frequency of microbubbles: Effect of viscosity. J. Acoust. Soc. Am. 2004, 116, 1463-1473. [CrossRef]

31. Yount, D.E. A microscopic investigation of bubble formation nuclei. J. Acoust. Soc. Am. 1984, 76, 1511-1521. [CrossRef]

32. Blatteau, J.E. Gas nuclei, their origin, and their role in bubble formation. Aviation Space Environ. Med. 2006, $77,1068-1076$.

33. Gateau, J.; Taccoen, N.; Tanter, M.; Aubry, J.F. Statistics of acoustically induced bubble-nucleation events in in vitro blood: A feasibility study. Ultrasound Med. Biol. 2013, 39, 1812-1825. [CrossRef]

34. Gateau, J.; Aubry, J.F.; Chauvet, D.; Boch, A.L.; Fink, M.; Tanter, M. In vivo bubble nucleation probability in sheep brain tissue. Phys. Med. Biol. 2011, 56, 7001-7015. [CrossRef]

35. Webb, I.R.; Payne, S.J.; Coussios, C.C. The effect of temperature and viscoelasticity on cavitation dynamics during ultrasonic ablation. J. Acoust. Soc. Am. 2011, 130, 3458-3466. [CrossRef] [PubMed]

36. Tung, Y.S.; Choi, J.J.; Baseri, B.; Konofagou, E.E. Identifying the inertial cavitation threshold and skull effects in a vessel phantom using focused ultrasound and microbubbles. Ultrasound Med. Biol. 2010, 36, 840-852. [CrossRef]

37. Guédra, M.; Inserra, C.; Gilles, B. Accompanying the frequency shift of the nonlinear resonance of a gas bubble using a dual-frequency excitation. Ultrason. Sonochem. 2017, 38, 298-305. [CrossRef] [PubMed]

38. Avvaru, B.; Pandit, A.B. Experimental investigation of cavitational bubble dynamics under multi-frequency system. Ultrason. Sonochem. 2008, 15, 578-589. [CrossRef] [PubMed]

39. Maxwell, A.D.; Cain, C.A.; Hall, T.L.; Fowlkes, J.B.; Xu, Z. Probability of cavitation for single ultrasound pulses applied to tissues and tissue-mimicking materials. Ultrasound Med. Biol. 2013, 39, 449-465. [CrossRef] [PubMed]

40. Vlaisavljevich, E.; Aydin, O.; Lin, K.W.; Durmaz, Y.Y.; Fowlkes, B.; ElSayed, M.; Xu, Z. The role of positive and negative pressure on cavitation nucleation in nanodroplet-mediated histotripsy. Phys. Med. Biol. 2015, 61, 663-682. [CrossRef]

41. Vlaisavljevich, E.; Xu, Z.; Maxwell, A.D.; Mancia, L.; Zhang, X.; Lin, K.W.; Duryea, A.; Sukovich, J.; Hall, T.; Johnsen, E.; et al. Effects of temperature on the histotripsy intrinsic threshold for cavitation. IEEE Trans. Ultrason. Ferroelectr. Freq. Control 2016, 63, 1064-1077. [CrossRef]

42. Yasuda, J.; Asai, A.; Yoshizawa, S.; Umemura, S.I. Efficient generation of cavitation bubbles in gel phantom by ultrasound exposure with negative-followed by positive-peak-pressure-emphasized waves. Jpn. J. Appl. Phys. 2013, 52, 07HF11. [CrossRef]

43. Behnia, S.; Sojahrood, A.J.; Soltanpoor, W.; Jahanbakhsh, O. Suppressing chaotic oscillations of a spherical cavitation bubble through applying a periodic perturbation. Ultrason. Sonochem. 2009, 16, 502-511. [CrossRef] [PubMed]

44. Sadighi-Bonabi, R.; Lahiji, F.A.F.; Razeghi, F. The effect of viscosity, applied frequency and driven pressure on the laser induced bubble luminescence in water-sulfuric acid mixtures. Phys. Lett. A 2016, 380, 2219-2226. [CrossRef]

45. Jung, S.; Kim, H. Hydrodynamic formation of a microlayer underneath a boiling bubble. Int. J Heat Mass Transf. 2018, 120, 1229-1240. [CrossRef]

(C) 2020 by the authors. Licensee MDPI, Basel, Switzerland. This article is an open access article distributed under the terms and conditions of the Creative Commons Attribution (CC BY) license (http://creativecommons.org/licenses/by/4.0/). 